Revista de

Estudios

Kantianos

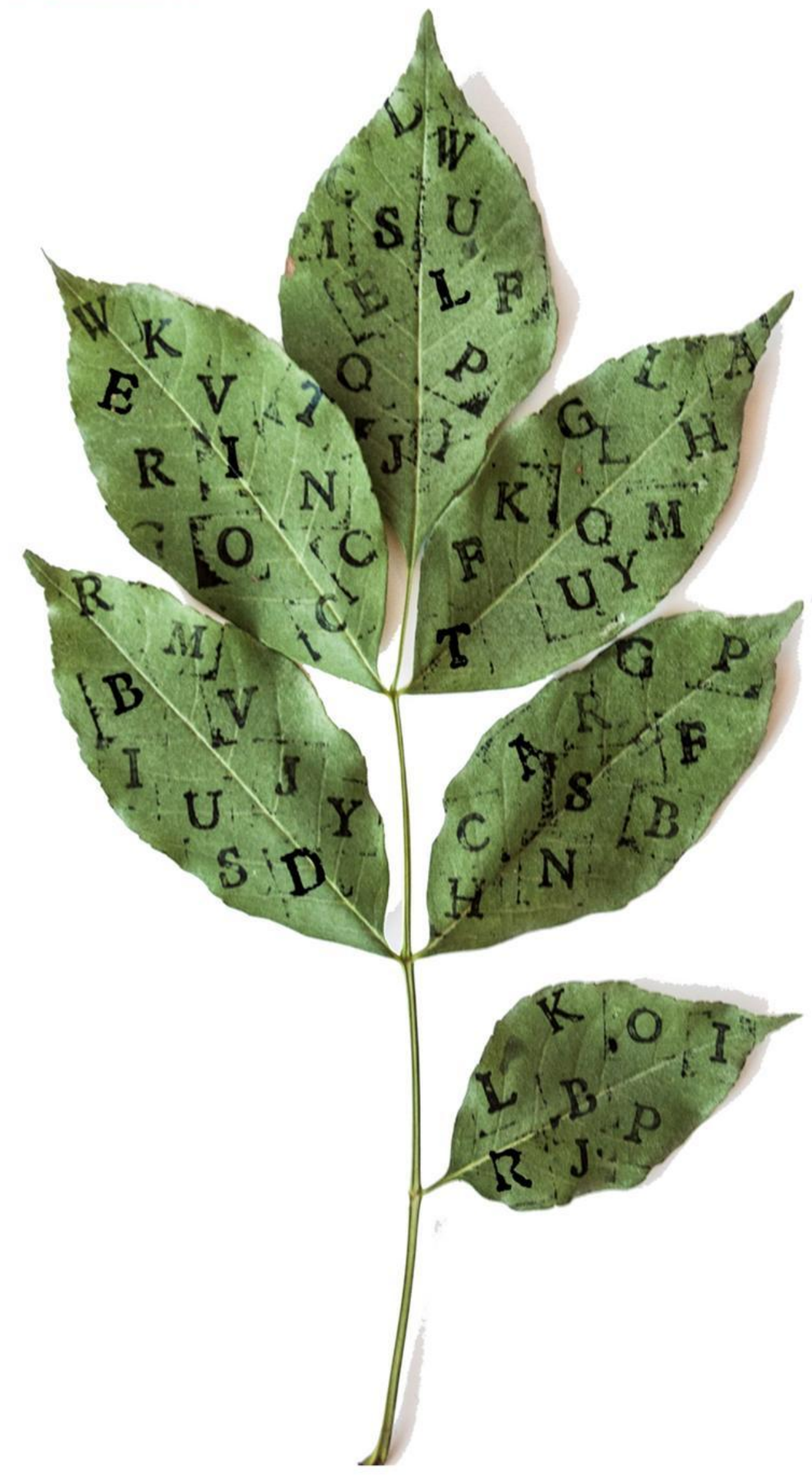


Revista de Estudios Kantianos 


\section{Revista de Estudios Kantianos}

Publicación internacional de la Sociedad de Estudios Kantianos en Lengua Española Internationale Zeitschrift der Gesellschaft für Kant-Studien in Spanischer Sprache International Journal of the Society of Kantian Studies in the Spanish Language

\section{Dirección}

Pedro Jesús Teruel, Universitat de València

pedro.teruel@uv.es

Hernán Pringe, CONICET-Universidad de Buenos Aires/

Universidad Diego Portales, Santiago de Chile

hpringe@gmail.com

\section{Secretario de edición}

Fernando Moledo, CONICET-Universidad de Buenos Aires

fernandomoledo@filo.uba.ar

\section{Secretaria de calidad}

Marcela García, Universidad de Morelia, México

garciar.marcela@gmail.com

\section{Editores científicos}

Jacinto Rivera de Rosales, UNED, Madrid

Claudia Jáuregui, Universidad de Buenos Aires

Vicente Durán, Pontificia Universidad Javeriana, Bogotá

Julio del Valle, Pontificia Universidad Católica del Perú, Lima

Jesús Conill, Universitat de València

Gustavo Leyva, Universidad Autónoma de México, México D. F.

María Xesús Vázquez Lobeiras, Universidade de Santiago de Compostela

Wilson Herrera, Universidad del Rosario, Bogotá

Pablo Oyarzun, Universidad de Chile, Santiago de Chile

Paula Órdenes Azúa, Universität Heidelberg 


\section{Comité científico}

Juan Arana, Universidad de Sevilla

Reinhardt Brandt, Philipps-Universität Marburg

Mario Caimi, Universidad de Buenos Aires

Monique Castillo, Université de Paris-Est

Adela Cortina, Universitat de València

Bernd Dörflinger, Universität Trier

Norbert Fischer, Universität Eichstätt-Ingolstadt

Miguel Giusti, Pontificia Universidad Católica del Perú

Dulce María Granja, Universidad Nacional Autónoma de México

Christian Hamm, Universidad Federal de Santa María, Brasil

Dietmar Heidemann, Université du Luxembourg

Otfried Höffe, Universität Tübingen

Claudio La Rocca, Università degli Studi di Genova

Juan Manuel Navarro Cordón, Universidad Complutense, Madrid

Carlos Pereda, Universidad Nacional Autónoma de México

Gustavo Pereira, Universidad de la República, Uruguay

Ubirajara Rancan de Azevedo, Universidade Estadual Paulista, Brasil

Margit Ruffing, Johannes Gutenberg-Universität Mainz

Gustavo Sarmiento, Universidad Simón Bolívar, Venezuela

Sergio Sevilla, Universitat de València

Roberto Torretti, Universidad Diego Portales, Santiago de Chile

Violetta Waibel, Universität Wien

Howard Williams, University of Aberystwyth

Allen W. Wood, Indiana University

Diseño, editor de estilo y maqueta

Josefa Ros Velasco, Universidad Complutense de Madrid

\section{Entidades colaboradoras}

Sociedad de Estudios Kantianos en Lengua Española (SEKLE)

Departament de Filosofia de la Universitat de València

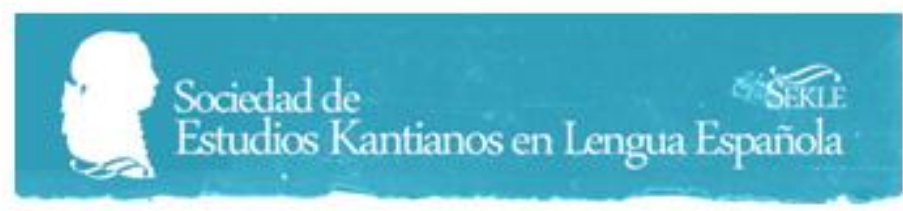




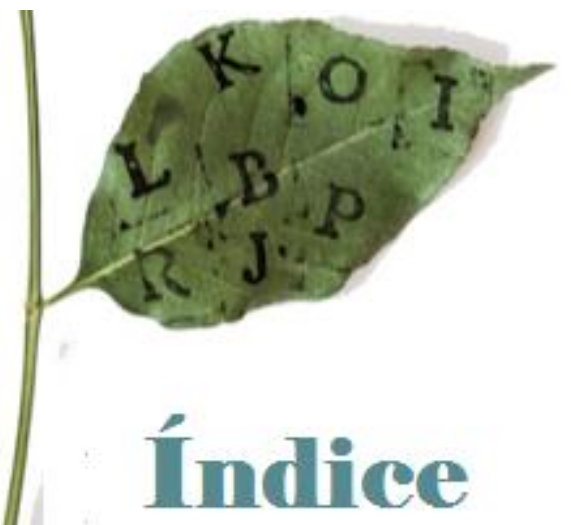

\section{Artículos}

9 Pensar como operación - Acerca de los presupuestos e implicaciones de la lógica formal moderna

Max Gottschlich

DOI 10.7203/REK.2.1.10059

20 Los conceptos kantianos de "facultad" y de "mente" frente a la lectura epigenética Antonino Falduto

DOI 10.7203/REK.2.1.10011

29 Arqueología filosófica y hermenéutica. Apuntes sobre la interpretación de la historia de la filosofía en Kant

José García Gómez del Valle

DOI 10.7203/REK.2.1.8811

48 Continuidad y novedad en la concepción histórica de Kant en El conflicto de las facultades

Natalia Lerussi

DOI 10.7203/REK.2.1.9892

60 It's impossible to will to be punished? Exploring consensual way out of the Kantian dilema Matías Parmigiani

DOI 10.7203/REK.2.1.8813

\section{Recensiones}

89 Immanuel Kant (trad. Alba Jiménez): Lecciones de filosofía moral Mrongovius II.

Salamanca, Sígueme, 2017, 160 pp. ISBN: 978-8-4301-1954-7

Pablo Veraza Tonda

DOI 10.7203/REK.2.1.9990 
91 Faviola Rivera Castro: Virtud, Felicidad y Religión en la Filosofía Moral de Kant. México, Instituto de Investigaciones Filosóficas-UNAM, 2014, 328 pp. ISBN: 978-607-02-4788-0

Gustavo Leyva

DOI 10.7203/REK.2.1.9992

93 Vicente de Haro Romo: Duty, Virtue and Practical Reason in Kant's Metaphysics of Morals. Hildesheim/Zürich/New York, Georg Olms Verlag, 2015, 342 pp. ISBN: 978-3487-15348-3

Eduardo E. Charpenel

DOI 10.7203/REK.2.1.9998

96 Paolo Grillenzoni: Kant e la scienza (1755-1760). Roma, Aracne, 2016, 575 pp. ISBN: 9788-854-89523-2

Daniele Savino

DOI 10.7203/REK.2.1.10054

100 Nuria Sánchez Madrid: A Civilização como destino. Kant e as formas da reflexão.

Florianópolis, Nefiponline, 2016, 288 pp. ISBN: 978-85-99608-16-6

Josefa Ros Velasco

DOI 10.7203/REK.2.1.9801

103 Laura Anna Macor: Die Bestimmung des Menschen (1748-1800): Eine Begriffsgeschichte. Forschungen und Materialien zur deutschen Aufklärung. Abteilung II: MonographienFMDA II, 25. Stuttgart-Bad Cannstatt, Frommann-Holzboog, 2013, 432 pp. ISBN: 978-37728-2615-3

Daniel Schwab

DOI 10.7203/REK.2.1.9989

\section{Informes}

109 Rezension zur Internationalen Tagung "Teleologische Reflexion in Kants Philosophie" Anna Pickhan (geb. Brechtel)

DOI 10.7203/REK.2.1.9993

\section{Entrevistas}

112 Entrevista a Pablo Muchnik

Fernando Moledo

DOI 10.7203/REK.2.1.10064

\section{Convocatorias y normas para autores}

118 Kant en México

DOI 10.7203/REK.2.1.10085

119 II Premio Kant para Jóvenes Investigadores e Investigadoras DOI 10.7203/REK.2.1.10086 
120 IV Congreso Internacional de la SEKLE, Valencia, 2018 DOI 10.7203/REK.2.1.10088

121 Normas para autores DOI 10.7203/REK.2.1.10091 


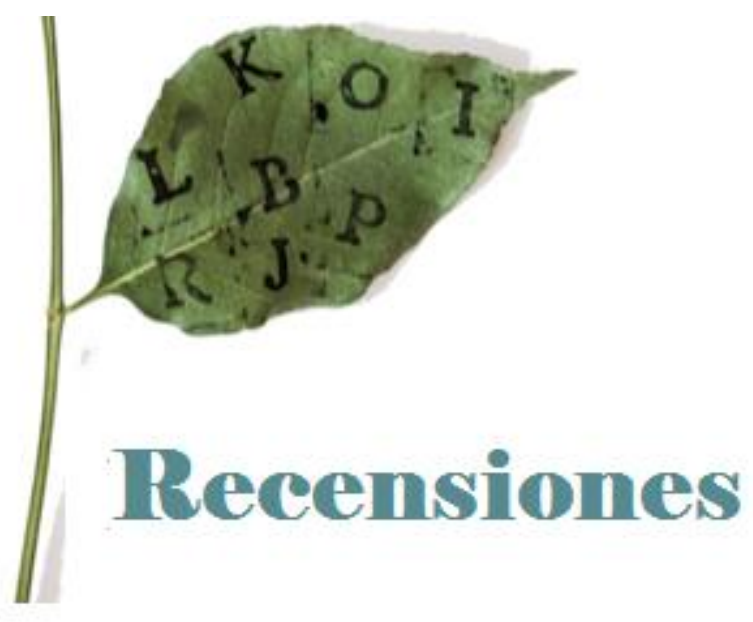




\title{
Nuria Sánchez Madrid: A civilização como destino. Kant e as formas da reflexão. Florianópolis, Nefiponline, 2016, 288 pp. ISBN: 978-85-99608- 16-6
}

\author{
JOSEFA Ros VELASCO ${ }^{1}$
}

La obra de Kant es una de aquellas que nunca dejan de arrojar sorpresas y que jamás termina de ser leída e interpretada; y precisamente por ello su recepción tampoco deja de crecer frente al paso del tiempo y a pesar de que los intereses filosóficos circulen en la actualidad por caminos que podrían parecer contrarios o lejanos al pensamiento kantiano. El monográfico en portugués A civilização como destino. Kant e as formas da reflexão [La civilización como destino. Kant y las formas de la reflexión], publicado en 2016 de la mano de la editorial brasileña Nefiponline, de la Profesora Dr. Nuria Sánchez Madrid (Universidad Complutense de Madrid) es un ejemplo de relectura y redefinición del corpus kantiano frente a las interpretaciones tradicionales que circunscriben la filosofía kantiana a la metafísica en su sentido más canónico. Esta obra recoge algunos de los textos de la autora publicados entre los años 2012-2017 y otros tantos inéditos en los que analiza la unión entre los presupuestos trascendentales kantianos y las emociones y los sentimientos con relación al proceso de desarrollo de la civilización humana desde un punto de vista antropológico.

La civilización como destino es una obra que nace en el seno del proyecto de investigación Poetics of Selfhood: memory, imagination and narrativity (CFUL, Fundação para a Ciência e a Tecnologia del Gobierno de Portugal), y de los grupos Naturaleza humana y comunidad (III). ¿Actualidad del humanismo e intelectualidad del hombre? y Retóricas del Clasicismo. Los puntos de vista (contextos, premisas, mentalidades) (MINECO). Su contenido empezó a gestarse durante una estancia que Sánchez Madrid realizó en la Faculdade de Filosofia e Ciências da UNESP (2012), y quizá tuvo ocasión de perfilarse durante 2014-2015, durante una estancia en el Instituto de Filosofía del CSIC. Pertenecen a aquellos años gran parte de los textos que compendia ahora en este volumen y que habían sido publicados previamente como artículos o capítulos de libro: «Filosofía, tom e ilusão musical em Kant. Da vivificação sonora do ânimo à recepção do tom da razão» (Trans/Form/Ação, 2012); «Maneira e método. Notas para uma genealogia da Crítica a partir da "Metodologia do gosto" da Crítica do Juízo» (Studia Kantiana, 2012); «Dos obstáculos da natureza aos obstáculos da razão. Uma leitura das «Preleções de pedagogia» de Kant em seis passos» (Kant e-prints, 2010); «Razão, consciência e ficção: J.-J. Rousseau nas Vorlesungen über Anthropologie de I. Kant», (Dissertatio,2014); «Corpo, desejo e razão: a sedução como arte de dominação na Antropologia de Kant» (Comentário da Antropologia em sentido pragmático de I. Kant, 2014); «Decorum e sociedade civil: Kant em diálogo com B. Gracián e Chr. Thomasius» (Investigações kantianas I: um debate plural, 2014); y, finalmente, «Kant e Freud sobre os fundamentos da obediência» (Ethic@, 2012).

Dos contribuciones, sin embargo, son completamente inéditas y sólo pueden encontrarse en el monográfico La civilización como destino. Nos referimos al capítulo 1, «A "Técnica da natureza": A co-originariedade crítica de arte, natureza e razão», y al capítulo 7, «A normatividade pragmática na Antropologia de Kant», además de la «Introducción» que brinda la propia autora. A estos inéditos se suman un «Prefacio» del Prof. Dr. Leonel Ribeiro dos Santos, del Centro de Filosofía de la Universidad de Lisboa, y un «Posfacio», del Prof. Dr. Márcio Suzuki, de la Facultad de Filosofía, Letras y Ciencias Humanas de la Universidad de São Paulo de Brasil, que revisan los puntos cardinales del volumen de la Dr. Sánchez Madrid. Este cómputo de textos que damos paso a revisar en mayor profundidad hace de La civilización como destino un lugar de máximo interés para todo aquél interesado por la filosofía contemporánea y la interpretación más reciente de la filosofía kantiana.

\footnotetext{
${ }^{1}$ Universidad Complutense de Madrid. Departamento de Historia de la Filosofía, Estética y Teoría del conocimiento.
} 
«As formas da reflexão e a genealogia da ordem» es el primer capítulode la obra de la Dr. Sánchez Madrid y está compuesto por los subapartados"Técnica da natureza": A co-originariedade crítica de arte, natureza e razão;Filosofia, tom e música em Kant: Vivificação sonora do ânimo e recepção do tom da razão; y, por último, Maneira e método: Notas para uma genealogia da Crítica a partir da "Metodologia do gosto" da Crítica do Juízo. En el primer subapartado, la autora propone un paralelismo entre la percepción musical y la búsqueda conceptual del que se desprende la idea de que la filosofía es capaz de arrojar luz cuando se adentra en la investigación de conceptos indeterminados, así como la música sugiere al oyente un determinado orden composicional a partir de la tonalidad. Este ejercicio no trata sino de establecer una heurística que posibilita el paso de las ideas reguladoras de la Crítica de la razón pura a los juicios reflexionantes estéticos y teleológicos de la Crítica del Juicio. Serán nombres de referencia, en este primer capítulo, los de Gilles Deleuze y Gérard Lebrun. El segundo subapartado es, de alguna manera, una continuación del primero. Filosofía, tom e ilusão musical em Kant, propone que la música es el arte más apto para captar los sentimientos que confieren sentido y proporción al mundo de la vida. Se trata de un arte de carácter físiológico, explica Sánchez Madrid, que a un mismo tiempo se entrelaza con la razón. En este punto, la autora aunará los presupuestos kantianos con los schopenhauerianos. Este capítulo termina de clarificar lo expuesto en el primero y advierte de la línea que seguirán los demás, mediante la exposición de que el sentimiento no se separa de la razón. Será ya en el tercer apartado en el que se manifieste de manera innegable que el método filosófico está ligado al modus aestheticus, así como la comunicabilidad del juicio del gusto sirve al proyecto de ilustración del público general.

El segundo capítulo de la obra se titula «Paradoxos da cultura: educação, consciência e diferença sexual» y se encuentra, como el anterior, dividido en tres subapartados: Dos obstáculos da natureza aos obstáculos da razão: Uma leitura das «Preleções de pedagogia» de Kant em seis passos Razão, consciência e ficção: J.-J. Rousseau nas Vorlesungen über Anthropologie de I. Kant; y Corpo, desejo e razão: a sedução como arte de dominação na Antropologia de Kant. Como apreciamos en un primer acercamiento, esta segunda parte del libro enfrenta tres temas que conjugan no sin dificultades: la pedagogía, la relación entre la conciencia, la razón y la ficción, y la cuestión de la diferencia sexual. Dos obstáculos da natureza aos obstáculos da razão y Razão, consciência e ficção, se adentran en la comunión posible entre la pedagogía y la antropología pragmática, partiendo de la analogía entre el concepto de educación en Kant y el pensamiento nietzscheano. Así las cosas, Sánchez Madrid pone de manifiesto que la educación no puede ser el resultado de modelos, toda vez que el individuo alcanza la autonomía a través del error y la transgresión. Corpo, desejo e razão, por su parte, aborda las diferencias entre los sexos desde el punto de vista de dos polos inseparables: el jurídico y el antropológico. Aunque la combinación de ambos resulta aparentemente conflictiva, desembocará de nuevo a la unión entre los afectos y la razón.

El tercer y último capítulo, «Os alicerces da civilização: a sociabilidade como aprendizagem da coação», discute el problema de la idea de normatividad en la antropología -y aquí enlaza con el capítulo anterior-. A lo largo de los apartados A normatividade pragmática na Antropologia de Kant; Decorum e sociedade civil: Kant em diálogo com B. Gracián e Chr. Thomasius; y Kant e Freud sobre os fundamentos da obediência, se presentan diferentes modalidades de articulación entre la antropología pragmática y moral en el sistema kantiano, a partir de la explicación de lo que conforma la prudencia mundana. La Dr. Sánchez Madrid tomará como punto de partida a Baltasar Gracián y a Christian Thomasius para reconstruir los temas del decoro y de la diferencia entre los deberes perfectos e imperfectos. Todo ello nos conduce a la clarificación de la idea de sociabilidad y del refinamiento cortesano propio de la Ilustración alemana. El cierre del libro, de la mano del subapartado que pone en relación a Kant y a Freud, hace las veces de una conclusión al volumen en su totalidad. En este último punto, la autora reconoce que Kant había hecho alusión a lo que Freud consideraba las pulsiones egoístas que afectaban negativamente a la vida colectiva. Así las cosas, observamos una anticipación de El malestar en la cultura en la filosofía kantiana que sólo Sánchez Madrid ha sabido abordar desde una posición distanciada de las interpretaciones tradicionales del pensamiento de Kant.

Es precisamente este ejercicio el que confiere a la obra de Nuria Sánchez Madrid un hilo conductor capaz de mostrar la vigencia de los planteamientos del ilustrado, aunando temas propios de la filosofía política y moral, de la estética y de la antropología, y poniendo sobre la mesa algunos de los tópicos que han sido desatendidos por los investigadores y desde los que se justifica una nueva 
lectura del corpus filosófico del pensador de Königsberg. A nadie extrañará, tras la lectura de esta obra, que una especialista como la Prof. Dr. Nuria Sánchez Madrid, que no sólo ha enriquecido el paradigma de recepción de la obra kantiana con sus aportaciones, sino que, además, colabora activamente para que otros tengan la oportunidad de hacerlo, haya sido capaz de ver lo que para otros ha quedado en la sombra: la vigencia del pensamiento kantiano y la importancia de su relectura y reinterpretación a la luz de las cuestiones que más preocupan a la sociedad contemporánea. 